\title{
Proposta di metodo semiquantitavo per la valutazione dell'efficacia della manutenzione di un pozzo
}

\author{
Nicola Cempini \\ Ingegnerie Toscane srl. \\ n.cempini@ingegnerietoscane.net
}

La manutenzione dei pozzi per acqua a servizio del servizi idrico integrato, quindi destinati all'alimentazione umana, è argomento di grande interesse sia dal punto di vista tecnico che dal punto di vista economico.

La produzione di acqua potabile di molti gestori italiani di acquedotti infatti, soprattutto nel centro nord, deriva dall'emungimento di acque sotterranee tramite pozzi.

Molto spesso il gestore si trova di fronte ad una scelta: fare la manutenzione di un pozzo esistente o procedere all'esecuzione di una nuova opera? Il water well professional deve saper rispondere correttamente a questa domanda; deve valutare il costo della manutenzione in rapporto ai benefici attesi e confrontare questa prospettiva con costi e benefici dell'esecuzione di un nuovo pozzo.

Oltre a ciò si presenta anche il problema di autorizzazione alla nuova perforazione, che in molti casi viene risolto attribuendo al nuovo pozzo la qualifica di "manutenzione straordinaria”, se però realizzato nelle immediate vicinanze del vecchio.

Oggi la manutenzione dei pozzi per acqua può contare su metodi, macchine e prodotti chimici che consentono di raggiungere buoni risultati e non di rado si riesce a ripristinare l'opera a livelli produttivi simili a quelli iniziali; tuttavia la manutenzione efficace di un pozzo, proprio a causa del fatto che i metodi si sono raffinati, non è un operazione definibile semplicisticamente a basso costo. D'altro canto la riperforazione di un pozzo è gravata dai costi di progettazione e direzione lavori, eventuale acquisizione del terreno, dall'iter tecnico amministrativo di autorizzazione sia in ambito ambientale che urbanistico e dall'iter di acquisizione del parere di idoneità in ambito igienico sanitario.

Manutenere o riperforare? Si tratta di un quesito che si presenta spesso al water well professional. La risposta deriva dall'analisi dello stato del pozzo, dal tipo di manutenzione necessaria e dai suoi costi, dalla complessità di procedere ad una nuova perforazione.

Non di rado poi tale quesito, nel caso degli acquedotti pubblici, non si limita ad un solo pozzo ma riguarda interi campi pozzi: il gestore spesso ha necessità di conoscere lo stato di quel particolare campo pozzi per poter programmare le manutenzioni e le riperforazioni e garantire la copertura finanziaria di progetti e lavori. Le manutenzioni possono essere solo di tipo meccanico oppure è necessario procedere anche ad una manutenzione chimica e magari a un ritubaggio (e ovviamente le due cose hanno costi diversi); la riperforazione può essere eseguita sia perforando effettivamente ex novo sia utilizzando uno dei metodi di estrazione delle aste e rialesa tura del perforo oggi disponibili.

In sintesi, da una parte c'è il concessionario e/o gestore che domanda: "che cosa devo fare dei miei pozzi? Quanti soldi devo stanziare per garantirne la funzionalità?”, dall'altra c'è un professionista che deve rispondere in modo rigoroso a questa domanda e che per farlo deve reperire tutte le informazioni disponibili sui pozzi in oggetto; deve eseguire le video ispezioni e delle prove speditive di portata, dopo di ché avrà a disposizione i dati necessari per una scelta.

Si insiste sulla necessità di eseguire la video ispezione del pozzo in quanto si tratta di una operazione di costo contenuto che se eseguita da personale esperto fornisce dati indispensabili per le successive decisioni; peraltro si ha un controllo diretto anche dell'effettiva corrispondenza tra i dati di completamento ed effettiva struttura dell'opera di captazione.

In questo articolo si propone un metodo per la valutazione della opportunità della manutenzione e della sua efficacia al fine di oggettivare per quanto possibile le informazioni basilari necessarie per rappresentare una guida orientativa per il professionista.

La manutenzione di un pozzo per acqua infatti soprattutto se destinato ad uso idropotabile, è una operazione che deve essere eseguita con metodo non prima di avere ottenuto alcune informazioni di base riguardanti il pozzo stesso e la sua storia operativa. Tutti gli water wells professional sanno che, perlomeno nel nostro Paese, non è infatti sempre detto che il gestore possieda le informazioni desiderate. Tuttavia, per decidere in modo appropriato che tipo di operazioni manutentive eseguire e se procedere alla manutenzione stessa un'idea della situazione bisogna farsela.

Nella fase ricognitiva occorre recuperare la stratigrafia, lo schema di completamento e intervistare il personale addetto in modo da avere informazioni sulla gestione e le problematiche che si sono manifestate nel tempo. E' molto utile anche l'esecuzione di una prova speditiva di portata da confrontare con le curve di collaudo originali.

Il metodo di seguito proposto si basa sul calcolo di due valori guida: valore guida $\alpha$ legato alle caratteristiche principali del pozzo e valore guida $\beta$ legato alle problematiche riscontrate.

Il primo, il valore guida alfa, si basa su 5 parametri guida a cui viene assegnato un punteggio sulla base di tre driver $(0,01-0,05-0,1)$ che vengono sommati secondo lo schema sottostante 


\begin{tabular}{|c|c|c|c|c|}
\hline \multicolumn{1}{|l|}{$\boldsymbol{\alpha}$} & parametri di pozzo & driver 0,01 & driver 0,05 & driver 0,1 \\
\hline 1 & tipo materiale & $\begin{array}{c}\text { acciaio bitumato o zincato spessore insufficiente, } \\
\text { acciaio inox spessore insufficiente }\end{array}$ & $\begin{array}{c}\text { acciaio bitumato o zincato spessore } \\
\text { sufficiente, termoplastico }\end{array}$ & \begin{tabular}{c} 
acciaio inox \\
\hline 2
\end{tabular} \\
\hline 3 & età (anni) & $>17$ & $12 \div 17$ & $<12$ \\
\hline 4 & regolarità manutenzione & insufficiente & mediocre & sufficiente \\
\hline 5 & profondità (m) & $>120$ & $50 \div 120$ & $<50$ \\
\hline
\end{tabular}

Come si intuisce facilmente, in questo caso un valore più elevato di $\alpha$ significa una stato "intrinseco" del pozzo migliore. I valori limite sono 0,05 e 0,5 che corrispondono rispettivamente ad un pozzo già in servizio da anni, gestito in modo superficiale e senza grande attenzione, sul quale sono stati fatti pochi interventi di manutenzione e magari poco adeguati a che ha perso oltre il $50 \%$ dell'efficienza originale e a un pozzo "giovane", realizzato con materiale adeguato, gestito bene ed ancora efficiente.

L'assegnazione dei diversi parametri segue le seguenti considerazioni:

- età: si presume che un pozzo realizzato di recente sia stato perforato seguendo criteri di assicurazione di qualità, non conosciuti all'epoca di una vecchia esecuzione;

- regolarità manutenzione: si deve desumere dalle informazioni reperite;

- profondità: si basa sul concetto che è più semplice e più economico manutenere un pozzo meno profondo rispetto a uno più profondo;

- efficienza residua: se ci sono prove di portata nel tempo, eseguite a valle delle manutenzione, è altrettanto importante reperirle e studiarle e in ogni caso valutare l'eventuale diminuzione della portata specifica rispetto al collaudo.

Il secondo valore guida $\beta$ si basa su 7 parametri guida a cui viene assegnato un punteggio sulla base di tre driver a cui è stato attribuito un punteggio maggiore rispetto ai precedenti $(0,01-0,07-0,12)$ con tre driver specifici per il parametro $\mathrm{n}$. 6 - fuoriuscita dreno $(0,01-0,4-0,8)$. La scelta di assegnare a questo parametro driver con punteggio maggiore dipende dal fatto che nella maggior parte dei casi osservare un fenomeno quale la fuoriuscita di dreno significa avere a che fare con un guasto veramente molto difficile da gestire. Lo schema è il seguente:

\begin{tabular}{|c|c|c|c|c|}
\hline $\boldsymbol{\beta}$ & $\begin{array}{l}\text { problematiche } \\
\text { riscontrate }\end{array}$ & driver 0,01 & driver $0,07(\mathrm{n} .6=0,4)$ & driver $0,12(\mathrm{n} .6=0,8)$ \\
\hline 1 & $\begin{array}{l}\text { ostruzioni } \\
\text { fisiche }\end{array}$ & nessuna, incipienti & presenti con ostruzione $<30 \%$ & presenti con ostruzione $>30 \%$ \\
\hline 2 & $\begin{array}{l}\text { ostruzioni } \\
\text { chimiche }\end{array}$ & nessuna, incipienti & $\begin{array}{l}\text { presenti molli - dure con ostruzione }< \\
30 \%\end{array}$ & presenti dure con ostruzione $>30 \%$ \\
\hline 3 & $\begin{array}{l}\text { ostruzioni } \\
\text { biologiche }\end{array}$ & nessuna, incipienti & $\begin{array}{c}\text { presenti molli - dure con ostruzione }< \\
30 \%\end{array}$ & presenti dure con ostruzione $>30 \%$ \\
\hline 4 & $\begin{array}{c}\text { lesioni da } \\
\text { correnti vaganti }\end{array}$ & nessuna, incipienti & leggere senza fuoriuscita dreno & forti con fuoriuscita dreno \\
\hline 5 & $\begin{array}{l}\text { corrosione o } \\
\text { rotture }\end{array}$ & $\begin{array}{l}\text { nessuna, incipienti, } \\
\text { saldature integre }\end{array}$ & $\begin{array}{l}\text { leggera, saldature leggermente corrose } \\
\text { (si può utilizzare questo driver se non c'è } \\
\text { nessuna fuoriuscita dreno) }\end{array}$ & $\begin{array}{l}\text { forte, saldature fortemente corrose (si deve } \\
\text { utilizzare questo driver se c'è fuoriuscita } \\
\text { dreno) }\end{array}$ \\
\hline 6 & $\begin{array}{l}\text { fuoriuscita } \\
\text { dreno }\end{array}$ & $\begin{array}{l}\text { nessuna - sospetta non } \\
\text { confermata }\end{array}$ & leggera - confermata da log tv & forte - guasto pompa \\
\hline 7 & $\begin{array}{l}\text { trascinamento } \\
\text { materiali fini }\end{array}$ & $\begin{array}{l}\text { nessuno - acqua } \\
\text { leggermente } \\
\text { opalescente }\end{array}$ & $\begin{array}{l}\text { acqua opalescente - presenza di granelli } \\
\text { di sabbia }\end{array}$ & $\begin{array}{l}\text { trascinamento sabbia e altri materiali fini tale } \\
\text { da non consentire l'uso ordinario }\end{array}$ \\
\hline
\end{tabular}

- ostruzioni fisiche: intasamento da parte degli elementi a granulometria più fine dell'acquifero;

- ostruzioni chimiche: principalmente ossidi di ferro e manganese, carbonato di calcio;

- ostruzioni biologiche: biofilm; colonie di ferrobatteri, batteri riduttori di solfati, coliformi;

- lesioni da correnti vaganti: caratteristici "buchi” di dimensioni centimetriche;

- corrosioni o rotture: corrosione galvanica; rotture meccaniche del tubo e/o dei filtri;

- fuoriuscita dreno: presenza di dreno nella succhiarola della pompa; pompa bruciata.

- trascinamento materiali fini: materiali fini in sospensione 
Una volta assegnato il driver che si ritiene opportuno ad ognuno dei parametri guida basterà sommare i punteggi per ottenere il seguente schema di valutazione proposto:

\begin{tabular}{|c|c|c|}
\hline classe & $\alpha / \beta$ & Probabilità di recupero pozzo \\
\hline 1 & $<0,5$ & $<25 \%$ \\
\hline 2 & $0,5 \div 0,7$ & $25 \% \div 50 \%$ \\
\hline 3 & $0,7 \div 1$ & $50 \% \div 75 \%$ \\
\hline 4 & $>1$ & $>75 \%$ \\
\hline
\end{tabular}

E' inoltre necessario eseguire una analisi sia chimica che batteriologica dell'acqua in modo da valutarne l'aggressività e/o il potere incrostante; sulla base di tutti questi dati il water well professional non solo sarà in grado di valutare la probabilità di riuscita della manutenzione, ma anche di proporre un piano di manutenzione programmata.

Questo metodo certamente non sostituisce l'esperienza del water wells professional ma ne integra la capacità di giudizio e soprattutto mira a fornire una indicazione semi-quantitativa che, soprattutto nel caso di grandi pianificazioni (es. piani di ambito), può rappresentare un valido aiuto nell'indirizzo delle risorse finanziarie.

I valori dei driver sul singolo parametro possono inoltre aiutare a decidere il tipo di intervento più idoneo, come nell'esempio di seguito illustrato:

$$
\begin{aligned}
& \alpha_{1}=0,05-0,1 \\
& \beta_{1}=0,07-0,12 \\
& \beta_{7}=0,07-0,12
\end{aligned}
$$

$$
\begin{aligned}
& \alpha_{1}=0,05-0,1 \\
& \alpha_{2}=0,05-0,1 \\
& \beta_{2}=0,07 \\
& \beta_{3}=0,07
\end{aligned}
$$

Pozzo di materiale adeguato, pochissimo o per niente corroso, senza rotture, con perdita di efficienza anche totale. Problematica principale: intasamento da trascinamento materiale fine, da limo a sabbia, dovuto con ogni probabilità a gestione scorretta. Intervento di manutenzione di tipo meccanico: pistonaggio; jetting tool; hydropuls, ad alta energia. Eventuale lavaggio con polifosfati. Esecuzione di nuovo sviluppo e prova speditiva di portata. Eventuale ripetizione fino al recupero

Pozzo di materiale adeguato, pochissimo o per niente corroso, senza rotture, con perdita di efficienza anche totale. Problematica principale: intasamento di tipo chimico - biologico. Intervento di manutenzione di tipo meccanico - chimico: pistonaggio; jetting tool; hydropuls, spazzola, ad alta energia -spurgo - lavaggio chimico con soluzione acida abbinata a biodisperdente con agenti chelanti - pistonaggio; jetting tool; hydropuls, spazzola, ad alta energia -spurgo - procedimento di sanificazione - spurgo - collaudo. 